Memorias del VII Encuentro Nacional de Experiencias en la Enseñanza de la Biología y la Educación Ambiental y II Congreso Nacional de Investigación en la Enseñanza de la Biología

\title{
ANÁLISIS E INTERPRETACIÓN DE SPOTS CON CONTENIDO CIENTÍFICO COMO RECURSO PARA LA IMPLEMENTACIÓN DE UNA ESTRATEGIA DIDÁCTICA
}

\section{Martha Cecilia Betancur Taborda ${ }^{1}$}

\section{Resumen}

Esta propuesta se centra en el diseño e implementación de una estrategia didáctica desarrollada a partir del análisis e interpretación de contenidos e ideas de ciencias naturales encontrados en algunos spot publicitarios. El estudio parte del reconocimiento de diferentes anuncios que presentan contenidos científicos, para posteriormente observarlos con los niños con el propósito de comprobar si los conocimientos adquiridos por los estudiantes, en el marco de las clases de ciencias, les permiten ser críticos cuando observan un spot con contenido científico. Lo anterior permite realizar un contraste entre las ideas que genera el observar la publicidad en su cotidianidad y su incidencia en las concepciones de ciencia construidas por los estudiantes.

La población objeto de estudio son niños de quinto grado, jornada mañana de educación básica primaria del colegio José Martí IED ubicado en la ciudad de Bogotá.

Los spot con contenido científico escogidos, fueron analizados e interpretados tanto por expertos en cada una de las disciplinas como por los niños, los resultados arrojados fueron el punto de partida para el diseño y aplicación de una

\footnotetext{
${ }^{1}$ Magister en didáctica de las ciencias Especialista en educación y gestión ambiental Licenciada en biología led Jose Martí Sed Bogota Grupo De Investigación Galatea macebeta@hotmail.com
} 
Bio-grafía Escritos sobre la Biologia y su Enseñanza.

Edición Extra-Ordinaria. ISSN 2027-1034 P.p 564 - 581

Memorias del VII Encuentro Nacional de Experiencias en la Enseñanza de la Biología y la Educación Ambiental y II Congreso Nacional de Investigación en la Enseñanza de la Biología

unidad didáctica sobre el concepto de energía en este caso obedece al análisis de los contenidos de ciencia encontrados en el spot que es uff "un cereal".

Palabras claves: spot, estrategia didáctica, aprendizaje y energía.

\section{Abstrac}

Thisproposal focuses onthe design and implementationofa teaching strategydeveloped from theanalysis and interpretation ofcontent and ideasofsciencefoundin someadvertising spots. The studyon the recognitionofdifferent adsthat presentscientific content, and later watchwith childrenin order tocheckwhether the knowledge acquiredby studentsas partofscience classes, allow them to becriticalwhen youobserve aspotwithscientific content. Thisallows fora contrast betweenthe ideas generated byobservingadvertising in yourdaily life andits impact onthe conceptions ofsciencebuiltby students

The population understudy isfifth gradersmorning shiftofbasic primaryschooleducationJoséMartílED located inthe city ofBogota.

Thechosenspotwithscientific content, were analyzed and interpretedbothby experts ineach of thedisciplinesaschildren, the results obtainedwerethe starting pointfor the design andimplementation ofa teaching uniton the conceptof energy inthis casedue to theanalysis of the contentsofsciencefound inspotisauff "cereal". Keywords: spot, teaching strategy, learningand energy

\section{Introducción}

La investigación parte de una situación problema que involucra la publicidad, en este caso los spot publicitarios que se transmiten a través de los medios de comunicación. El propósito, es la utilización de los spot con contenido científico como recurso para el diseño, construcción y aplicación de una estrategia didáctica 
Bio-grafía Escritos sobre la Biologia y su Enseñanza.

Edición Extra-Ordinaria. ISSN 2027-1034 P.p 564 - 581

Memorias del VII Encuentro Nacional de Experiencias en la Enseñanza de la Biología y la Educación Ambiental y II Congreso Nacional de Investigación en la Enseñanza de la Biología

para la enseñanza y el aprendizaje de las ciencias naturales, la cual es dirigida a los niños de quinto grado de educación básica primaria.

La propuesta se estructuró a la luz de diversos trabajos sobre la enseñanza y el aprendizaje de las ciencias naturales, como también estudios realizados en otros países sobre el análisis crítico de contenidos de ciencia mostrados en la publicidad y su posterior utilización como recurso para la implementación de estrategias didácticas. El trabajo muestra como aspecto innovador la didáctica propuesta y la población objeto de estudio, en este caso, los niños de quinto grado de educación básica primaria de la jornada mañana del Colegio José Martí, en edades comprendidas entre los 9 y 14 años.

El diseño de una estrategia didáctica que parta del análisis e interpretación de contenidos de ciencias naturales encontrados en algunos spot, implica la puesta en marcha de una metodología y de una serie de actividades para lograr un aprendizaje significativo en los niños, es así como se parte de la indagación de los intereses de ellos con respecto a la publicidad y específicamente los spot, es decir, se averiguó cuál es el tipo de publicidad que más les gusta observar.

Posteriormente y después de indagar el tipo de publicidad que más les llama la atención a los niños, se escogieron cinco spot publicitarios de acuerdo a los parámetros que ellos habían expresado en un instrumento cuestionario aplicado, también se tuvo en cuenta que en los contenidos mostraran expresiones o nociones relacionadas con temáticas propias de las ciencias naturales.

Los spot seleccionados se emitieron con frecuencia en la televisión colombiana durante los años 2010 y 2011, esta publicidad (spot) corresponde a los siguientes productos: qué es uff. "un Cereal", Detergente Ariel, Cepillo dental Colgate, Bisolvón, Isodinemint; los cuales fueron analizados por profesores expertos en las diferentes disciplinas de las ciencias y los niños. Los resultados arrojados del 
Memorias del VII Encuentro Nacional de Experiencias en la Enseñanza de la Biología y la Educación Ambiental y II Congreso Nacional de Investigación en la Enseñanza de la Biología

análisis de expertos y niños dieron paso al diseño y aplicación de una unidad didáctica sobre el concepto de energía como parte de la estrategia propuesta.

La estrategia didáctica comprende dos Fases, la primera de ellas hace alusión a la escogencia, análisis e interpretación de spot con contenido científico tanto por doctores expertos en diversas disciplinas de las ciencias naturales como por los niños de quinto grado de educación básica primaria. La fase dos se refiere a la aplicación de una unidad didáctica relacionada con una de las temáticas presentadas en el spot publicitario en este caso sobre el concepto de la energía, la aplicación de la unidad didáctica posibilitó evaluar la estrategia didáctica, es decir, si los aprendizajes adquiridos en las clases de ciencias les permitieron a los niños ser críticos cuando observan o escuchan un spot con contenido científico.

La aplicación de la unidad didáctica posibilitó que los niños ganaran en comprensión de la temática propuesta, además brindó herramientas conceptuales para analizar spot con contenido científico. Por último se evaluó los conocimientos adquiridos por los niños en el marco de las clases de ciencias, teniendo en cuenta un proceso de regulación de aprendizajes que comprende la autoevaluación, la coevaluación y la heteroevaluación.

\section{Situación Problema}

Para la formulación del problema se parte de la siguiente premisa: los niños generalmente, presentan nociones con respecto a los conceptos científicos y fenómenos relacionados con las ciencias naturales, algunos de estos conocimientos los elaboran debido a la interacción con los medios de comunicación, presentándose con frecuencia errores conceptuales con fuerte resistencia al cambio; los cuales se evidencian en la forma como el niño se expresa con respecto a ciertos fenómenos naturales o problemáticas relacionadas con las ciencias naturales. 
Memorias del VII Encuentro Nacional de Experiencias en la Enseñanza de la Biología y la Educación Ambiental y II Congreso Nacional de Investigación en la Enseñanza de la Biología

La anterior situación implica un cambio en la enseñanza y el aprendizaje de las ciencias de manera que, se diseñen e implementen estrategias didácticas en el aula de clase en pro de la construcción y desarrollo de conocimientos científicos. Es así como por lo expuesto anteriormente, surge la pregunta ¿Qué elementos debe poseer una estrategia didáctica que parta del análisis de contenidos científicos encontrados en algunos spot publicitarios transmitidos por la televisión?

\section{Referentes Teóricos}

Las perspectivas teóricas consultadas en la presente investigación hacen referencia a la concepción de ciencia desde la teoría de la complejidad, a los modelos didácticos vinculados al trabajo, entre ellos la didáctica problémica y la fundamentación del concepto de unidad didáctica desde la mirada de Neus San martí, por último se hace alusión a la enseñanza del concepto de energía para los niños.

Concepción de ciencia: Bronowsky, citado por Morín (1994) afirma el concepto actual de ciencia no es "ni absoluto, ni eterno". Teniendo en cuenta lo anterior Morín toma de Vico el término de la scienza nuova para referirse a la ciencia como una modificación, una transformación, un enriquecimiento. La ciencia nueva, es simplemente algo cuyas consecuencias serán incalculables: no solamente el objeto debe ser adecuado a la ciencia, la ciencia debe también ser adecuada a su objeto.

Didáctica Problemica es uno de los pilares para el desarrollo de la unidad didáctica en tal sentido Bravo (2004) afirma:

El propósito de la didáctica problémica es problematizar el conocimiento y la cultura en la perspectiva de desarrollar instrumentos mentales y sistemas operacionales. Problematizar es comprender las dinámicas (dialéctica) del conocimiento desde las tensiones histórico-lógicas. Para él hay una aproximación estructural a la complejidad que ha alcanzado el conocimiento contemporáneo de 
Memorias del VII Encuentro Nacional de Experiencias en la Enseñanza de la Biología y la Educación Ambiental y II Congreso Nacional de Investigación en la Enseñanza de la Biología

los mundos físico-biótico y antrópico y con ello se resaltan los niveles de problematización del conocimiento así como la incapacidad de los paradigmas empírico tradicionales y positivistas para observar y comprender lo incierto, lo difuso y contradictorio; de esta manera se evidencia no solo la necesidad de la perspectiva interdisciplinaria- transdisciplinar, sino también los brotes de nuevas disciplinas transversales (p.190).

Estrategia Didáctica, Carrasco (2004) define la estrategia didáctica como aquellos enfoques y modos de actuar que hace que el profesor dirija el aprendizaje de los estudiantes, ellas se refieren a todos los actos favorecedores del aprendizaje, las estrategias didácticas facilitan a los estudiantes ser más competentes en la regulación de sus aprendizajes.

Unidad didáctica: Sanmartí (2002) afirma que la actividad didáctica es un conjunto de acciones planificadas por el profesorado que tienen como finalidad promover el aprendizaje de los estudiantes en relación con determinados contenidos, para la autora la profesión de enseñar se concreta en el diseño de unidades didácticas formadas por secuencias en las cuales hay unos objetivos de aprendizaje específicos.

Televisión y spot: la televisión y específicamente los spots publicitarios llevan implícitos ideas valores o productos, de ahí la importancia de saber integrar la televisión al aula y específicamente a la educación, Ferrés (1994) sostiene, que para una adecuada integración de la televisión al aula es necesario atender dos dimensiones formativas: educar en la televisión y educar con la televisión.

Fundamentación teórica sobre el concepto de energía: según Feynman citado por Gonzáles (2008) afirma lo siguiente sobre el concepto de la energía "Es importante notar que en la física de hoy día no tenemos conocimiento acerca de lo que es la energía. Es un algo abstracto en el sentido que no nos dice el mecanismo o las razones para las diversas fórmulas" (p. 1) de igual manera el 
Memorias del VII Encuentro Nacional de Experiencias en la Enseñanza de la Biología y la Educación Ambiental y II Congreso Nacional de Investigación en la Enseñanza de la Biología

autor plantea la dificultad de encontrar en los libros de texto una definición generalizada de energía que no pueda ser impugnada por una razón u otra.

\section{Metodología}

La investigación se estructuró en dos fases en las cuales se desarrolló un trabajo de corte cualitativo que sigue dos estrategias de indagación: una descriptiva participativa y estudio de caso, para la primera estrategia el producto de la investigación es netamente descriptivo, en donde se obtienen datos producto de las entrevistas y las actividades observables en el aula de clase. La segunda estrategia es el estudio de caso, utilizado para describir y analizar el proceso de construcción de conceptos científicos interiorizados por los niños a partir de la interpretación y el análisis de los contenidos de ciencia mostrados en los spot seleccionados y la posterior aplicación de una unidad didáctica.

\section{FASE I Análisis descriptivo e interpretación de spot con contenido científico,} esta fase contempla una estrategia de indagación descriptiva participativa, en donde se inicia con el estudio de la publicidad transmitida por la televisión colombiana en las franjas infantiles y juveniles durante los meses de junio a diciembre del año 2011.Posteriormente se hace una selección cuidadosa de los spot que presenten contenidos científicos y se someten al análisis e interpretación de doctores (expertos) a la luz de las teorías científicas que ellos manejan.

De otro lado se realiza una fundamentación teórica y metodológica con respecto a las visiones e ideas de ciencia que transmite la publicidad (específicamente los spot) y los principios teóricos que posteriormente le darán sustento a la investigación, también es clave el diseño y aplicación de un instrumento cuestionario para recoger información sobre el tipo de publicidad transmitida por la 
Memorias del VII Encuentro Nacional de Experiencias en la Enseñanza de la Biología y la Educación Ambiental y II Congreso Nacional de Investigación en la Enseñanza de la Biología

televisión colombiana que más le llama la atención a los niños de quinto grado de educación básica primaria del colegio José Martí jornada mañana.

El instrumento diseñado fue validado previamente por un experto, el Dr. en Educación Mauricio Lizarralde, quién contribuyó con sus observaciones a mejorar y ajustar el instrumento, posteriormente se realizó un primer pilotaje del cuestionario con un grupo de estudiantes de un grado equivalente al grupo de investigación, ellos dieron sus aportes al instrumento; por último, se aplicó al grupo objeto de estudio, se sistematizaron los datos y de acuerdo a los resultados se dio paso a la observación y escogencia de spot con contenido científico que más les llaman la atención a los niños del grado quinto y que son emitidos en las franjas infantil y juvenil de la televisión colombiana.

La publicidad seleccionada debía presentar contenidos de ciencia, en este caso se escogieron cinco spot publicitarios: qué es uff...un Cereal, Detergente Ariel, Cepillo dental Colgate, Bisolvón, Isodinemint, los cuales fueron analizados por doctores expertos en las áreas de Educación, Dr. Mauricio Lizarralde; en el área química el Dr. Carlos Javier Mosquera; en el área de física, la Magister Diana Luz López; en el área de antropología, el Dr. Julián Arturo y la Magister Olga palacio; comunicación y semiología Dr. Carles Casals Pascual, quienes previo análisis de los spot y la posterior realización de una entrevista (informal, espontánea y en grupo) emitieron sus visiones y conceptos a la luz de las teorías científicas que ellos manejan. Igualmente los niños del grado quinto de primaria a través de una entrevista expresaron su punto de vista con respecto a los contenidos e ideas de ciencia que transmitían los spot.

FASE II diseño y aplicación de la intervención didáctica, se aplica una estrategia de indagación, estudio de caso, en la cual se tiene en cuenta los resultados arrojados en la primera fase como punto de partida para el diseño y 
Memorias del VII Encuentro Nacional de Experiencias en la Enseñanza de la Biología y la Educación Ambiental y II Congreso Nacional de Investigación en la Enseñanza de la Biología

aplicación de dos instrumentos: uno de ellos de orden cuantitativo y el otro cualitativo; Estos contienen preguntas basadas en las ideas manifestadas en la entrevista sobre la interpretación que los niños realizaron sobre los spot. Al respecto Pérez (1994) afirma que "una de las formas de dar fiabilidad en los instrumentos consiste en elaborar una prueba con preguntas que sean equivalentes" (p.72).

Con los resultados y la información arrojada en los instrumentos se elabora una unidad didáctica como eje articulador de la investigación, tendiente a favorecer la comprensión de conceptos relacionados con las ciencias naturales y cuyo objetivo es que los niños adquieran una visión más próxima al conocimiento científico. En este caso la unidad didáctica propuesta se elabora desde la perspectiva de Sanmartí (2001), Couso et al (2.005 p.38) y Bravo (2004) sobre la temática de energía.

La temática sobre la energía se escogió debido a que está propuesta en el plan de estudios para el grado quinto, además los análisis derivados de la observación e interpretación de los contenidos de ciencia transmitidos por el spot que es uff "un cereal", conllevaron a adoptar este concepto para posteriormente desarrollarlo en la unidad didáctica planteada en esta fase.

En esta investigación la evaluación es un componente que permite la regulación de los aprendizajes, en este sentido Según Sanmartí (2002) se tiene en cuenta tres tipos de evaluación:

1. Autoevaluación: entendida como aquella que hacen los niños de su propio aprendizaje, en este caso el instrumento que se emplea es un KPSI 2. Coevaluación o regulación mutua: desarrollada por la interacción mutua entre los propios estudiantes, es decir, corresponde a la evaluación que le hace un par de las producciones que ellos realizan 
Bio-grafía Escritos sobre la Biologia y su Enseñanza.

Edición Extra-Ordinaria. ISSN 2027-1034 P.p 564 - 581

Memorias del VII Encuentro Nacional de Experiencias en la Enseñanza de la Biología y la Educación Ambiental y II Congreso Nacional de Investigación en la Enseñanza de la Biología

3. Heteroevaluación: la evaluación que le hace el profesor al niño de acuerdo a los criterios establecidos, el profesor detecta y regula lo que el estudiante no es capaz de regular por sí mismo.

\section{Análisis De Resultados}

Teniendo en cuenta las fases planteadas en la metodología y un proceso riguroso de observación, diseño, elaboración y aplicación de los instrumentos de recolección de la información durante las fases de la intervención, se puede afirmar que en nuestra sociedad y en especial en la población objeto de estudio la televisión es el medio de comunicación que más utilizan los niños, ellos se sienten cautivados por la "televisión" de ella obtienen información relevante y en ciertos casos útil para la compra de los productos que en ella se anuncian, bien sea para el consumo personal, del hogar o el uso de medicamentos en especial aquellos utilizados para mejorar la salud, en este caso para contrarrestar los resfriados y la tos.

En el estudio se evidencia que un $70 \%$ de los niños observan en promedio cuatro horas al día la televisión, es decir, unas 28 horas semanales durante el año, en contraste con el número de horas que pasan en el aula escolar, es decir, 25 horas semanales; los anteriores resultados abren un campo de trabajo e investigación sobre el origen social de ciertas concepciones de ciencia a partir de los mensajes publicitarios.

De otro lado, los resultados arrojados en el instrumento aplicado muestran que un $48 \%$ de los niños le prestan atención a la publicidad, en este caso, los spot publicitarios transmitidos por la televisión colombiana, los spot que más les llama la atención a los niños son los relacionados con juguetes, dibujos animados y 
Memorias del VII Encuentro Nacional de Experiencias en la Enseñanza de la Biología y la Educación Ambiental y II Congreso Nacional de Investigación en la

\section{Enseñanza de la Biología}

situaciones divertidas, muchos de ellos coincidieron en que la publicidad les enseña conceptos relacionados con las ciencias naturales.

Los Doctores expertos y los niños observaron, analizaron e interpretaron los spot contenido científico y posteriormente respondieron una entrevista de carácter informal, espontánea y en grupo. Las entrevistas se transcribieron conservando y respetando el pensamiento del interlocutor, sin transformarlo, la información arrojada se organizó en unidades de análisis que dieron lugar al establecimiento de categorías punto de partida para diseño, estructuración y aplicación de una unidad didáctica.

Es importante anotar que cada uno de los expertos hizo la interpretación de los spot a luz de sus conocimientos, notándose que los especialistas en el área de las ciencias naturales, enfatizaron en las posibles visiones de ciencia, contenidos y palabras relacionadas con esta disciplina, al igual que las distorsiones conceptuales que transmiten algunos spot analizados, ejemplo de ello, el caso del spot "uff es un cereal" en el que se confunde el concepto de fuerza con el de energía.

Algunas de las interpretaciones realizadas por los expertos las podemos evidenciar en el siguiente texto:

Voces de los expertos spot "QUÉES UFF UN CERERAL"

"Representación del científico como un súper héroe que soluciona un problema de falta de energía, se muestra la imagen de ciencia como panacea en donde hay i Iconos, representaciones, símbolos y gráficas sobre productos propios y originados del conocimiento Científico. Hay un Obstáculo para el aprendizaje, diversidad de concepciones sobre la energía, asocia la idea de energía con fuerza generando distorsión conceptual".

Voces de los niños spot "QUÉES UFF UN CERERAL" 
Bio-grafía Escritos sobre la Biologia y su Enseñanza.

Edición Extra-Ordinaria. ISSN 2027-1034 P.p 564 - 581

Memorias del VII Encuentro Nacional de Experiencias en la Enseñanza de la Biología y la Educación Ambiental y II Congreso Nacional de Investigación en la Enseñanza de la Biología

Se mencionan las palabras energía, vitaminas y fortaleza, cereal nutritivo.

Pretende Vender un cereal.

El niño no tiene la fuerza para levantar un carro, el cereal no nutre tanto como para levantar un carro. El cereal me gusta comprarlo porque tiene vitaminas, el cereal proporciona energía, el cereal da fuerza y el niño puede alzar el carro.

El spot nos enseña a ser fuertes, a comer cereal y cosas nutritivas y el niño después de comer el cereal puede alzar el carro.

Los expertos entrevistados concluyen que este tipo de publicidad se podría utilizar como un recurso para la enseñanza de las ciencias, en donde el docente podría realizar con los estudiantes un análisis de los contenidos mostrados e introducirlos a una temática relacionada con las ciencias naturales.

Los niños en sus respuestas hacían referencia a palabras y acciones mostradas de manera explícita en los spot y en muchas oportunidades se notaba en ellos una convicción muy general de creer en la veracidad del anuncio, ya que, algunos spot recurrían a afirmaciones como "científicamente comprobado", lo cual hace que los niños asuman estas expresiones como una verdad absoluta; esta situación comprueba lo que muchos autores como Campanario han afirmado," en muchas ocasiones la publicidad recurre al prestigio de la ciencia y la tecnología para lograr que sus productos sean comprados y consumidos".

Los resultados muestran que los niños al observar los spot y responder a las preguntas de la entrevista, en escasos momentos lograron discernir una visión de ciencia, exceptuando el caso del spot de Ariel, en donde aparecen varias personas vestidas de blanco manipulando artefactos, los estudiantes respondieron "la ciencia es avanzada, hay científicos investigando y vestidos de blanco" lo anterior evidencia que los niños construyen una imagen de ciencia transmitida por 
Memorias del VII Encuentro Nacional de Experiencias en la Enseñanza de la Biología y la Educación Ambiental y II Congreso Nacional de Investigación en la Enseñanza de la Biología

la publicidad que no corresponde a la construida social y culturalmente por la comunidad científica y la filosofía de la ciencia, a diferencia de los expertos los cuales al realizar el análisis de la publicidad establecieron muchas más concepciones deformadas de la ciencia transmitidas por los spot.

De acuerdo a los resultados anteriores se tomó el spot "que es uff...un cereal "con la finalidad de indagar que interpretación le dan los niños a este anuncio que presenta contenidos de ciencia. A partir de las ideas que ellos expresaron con respecto a lo que les transmite este spot, se inicia la aplicación de una unidad didáctica, relacionada en este caso el concepto de energía, lo cual permite realizar un posterior análisis y evaluación de los aprendizajes generados después de la intervención didáctica y así poder constatar si los conocimientos adquiridos en el marco de las clases de ciencias, les permite a los niños ser críticos cuando observan publicidad con contenido científico y si es posible discernir los errores conceptuales que a veces presenta la publicidad.

Con respecto a la evaluación y regulación del aprendizaje en esta propuesta se tiene en cuenta la lectura y firma de un contrato Didáctico, en el cual se les pide a los estudiantes cumplir con una serie de acuerdos y sobre todo llevar un registro en una libreta de ciencias como especie de diario de campo en el que deben escribir lo realizado en las actividades propuestas con respecto a lo que han aprendido y que se les ha dificultado. El segundo instrumento utilizado en esta propuesta es un KPSI en donde los niños reflexionan sobre lo que han aprendido y que dificultades han presentado, este instrumento fue exitoso pues permitió reflexionar y re significar otro tipo de actividades que ayudasen a los niños a la construcción de conocimientos.

Finalmente se concluye que la estrategia didáctica fue exitosa, ya que los niños realizaron un análisis crítico de los spot con contenidos científicos, 
Memorias del VII Encuentro Nacional de Experiencias en la Enseñanza de la Biología y la Educación Ambiental y II Congreso Nacional de Investigación en la Enseñanza de la Biología

específicamente, que es uff "un cereal", logrando discernir el error conceptual que presentaba éste; además utilizaron la información suministrada en la unidad didáctica para construir significados y conceptos con respecto a la temática de la energía.

\section{Conclusiones}

Acorde al tipo de investigación realizada, las conclusiones se muestran teniendo en cuenta los siguientes aspectos: al proceso de investigación y metodología, el análisis e interpretación de spot con contenido científico, la unidad didáctica sobre la temática de la energía, los procesos de regulación de los aprendizajes y los aprendizajes generados por los estudiantes.

4. El proceso de investigación cualitativa realizado y estructurado en las técnicas e instrumentos seleccionados, permitió una adecuada recolección de la información, que posteriormente posibilitó el análisis y el logro de los objetivos propuestos en la investigación.

5. Así mismo, es vital destacar la importancia del diseño y construcción de los instrumentos que permitieron una adecuada recolección, análisis y presentación de la información, otorgándole a la presente investigación la validez y confiabilidad necesaria en el campo de la Didáctica de las Ciencias.

6. En la fase I de la investigación los resultados del primer instrumento cuestionario muestran que los niños de quinto grado de educación básica primaria del colegio José Martí observan con frecuencia la televisión, además le prestan atención a la publicidad presentada de la cual obtienen información.

7. Cuando los niños observan por vez primera los spot hay coincidencia en que los anuncios hacen referencia a la ciencia, a científicos vestidos con bata blanca, lo cual permite afirmar que se transmite una concepción de ciencia 
Memorias del VII Encuentro Nacional de Experiencias en la Enseñanza de la Biología y la Educación Ambiental y II Congreso Nacional de Investigación en la Enseñanza de la Biología

como fuente de autoridad y verdad absoluta, dicha concepción es la que los niños adquieren y está totalmente opuesta a la realidad.

8. Al observar los estudiantes por primera vez el spot "que será uff... un cereal, se evidencia en ellos una confusión entre el concepto de fuerza y energía, dicho error conceptual lo transmite el spot.

9. El diseño de la unidad didáctica, soportado en actividades como lecturas, experiencias de conocimiento, resolución de situaciones problema, elaboración de mapas conceptuales y el análisis crítico de la publicidad, permitió a los niños la apropiación de aprendizajes relacionados con la energía, el fortalecimiento de la argumentación y el gran entusiasmo por las clases de ciencias, lo cual se refleja en los diarios de campo, apreciaciones y escritos de ellos.

10. El firmar un contrato didáctico, resolver los KPSI y participar en las actividades de coevaluación, reflejan la importancia y el compromiso de la regulación de los aprendizajes por parte de los niños.

11. Este trabajo permitió conocer en primera instancia como analizan los niños la publicidad con contenido científico, reflejando que ellos siempre hacen referencia a la palabra o el mensaje explicito encontrado en los spot. Después de aplicar la unidad didáctica los niños son capaces de encontrar los errores conceptuales transmitidos en la publicidad y realizar una reflexión desde el punto de vista de las ciencias naturales, es decir, presentan un acercamiento a lo expresado por los expertos y la literatura.

12. La aplicación y desarrollo de la unidad didáctica evidencia procesos de aprendizaje en los niños mostrando un enriquecimiento en sus ideas y lenguaje 
Memorias del VII Encuentro Nacional de Experiencias en la Enseñanza de la Biología y la Educación Ambiental y II Congreso Nacional de Investigación en la Enseñanza de la Biología

el cual se refleja en la adquisición con sentido del vocabulario propio de las ciencias naturales. También mostró un proceso continuo en el cual los estudiantes utilizaron adecuadamente la información suministrada para construir significados sobre la energía.

13. La aplicación de la unidad didáctica, el KPSI y las actividades de coevaluación permitió una valoración del trabajo en equipo y la discusión de ideas, (aproximación al trabajo realizado por las ciencias y los científicos) lo cual se constata en los diarios de campo realizados por ellos.

\section{Bibliografía}

1. Angulo, F. (2002). Aprender a enseñar ciencias: Análisis de una propuesta para la formación inicial del profesorado de secundaria, basada en la metacognición. Tesis doctoral, Universidad Autónoma de Barcelona. Disponible en: http://www.tesisenred.net/bitstream/handle/10803/4693/fad1de5.pdf?sequence=1 . [2010,5 marzo]

http://www.tesisenred.net/bitstream/handle/10803/4693/fad2de5.pdf?sequence=2 . [2010,5 marzo

2. Bonilla, E; Rodríguez, P (2005). Más allá del dilema de los métodos. Bogotá: Grupo editorial Norma.

3. Bravo, N. (2002).Pedagogía Problémica. Cali: Faid Editores.

4. Briones, G (1989).Métodos y técnicas de investigación aplicadas a la educación y a las ciencias sociales. Módulo 5. Bogotá: ICFES 
Bio-grafia Escritos sobre La Biología y su Enseñanza.

Edición Extra-Ordinaria. ISSN 2027-1034 P. p 564-581

Memorias del VII Encuentro Nacional de Experiencias en la Enseñanza de la Biología y la Educación Ambiental y II Congreso Nacional de Investigación en la Enseñanza de la Biología

5. Buckingham, D. (2005). Educación en medios. Barcelona: Paidos

6. Buch, T (1.999).Sistemas Tecnológicos. Argentina: Aique

7. Barnes, N. Curtis, H. Schnek, A. Massarini, A. (2008).Biología. Madrid: Editorial médica Panamericana. Disponible en:

14. Campanario, J. Moya, A. Otero, J. (2001).Invocaciones y usos inadecuados de la ciencia en la publicidad. Enseñanza de las ciencias, 45-46. disponible en:

http://www2.uah.es/jmc/an1.pdf .[2010,20 de noviembre]

15. Candela, A. (1991).Argumentación y conocimiento científico escolar. Infancia y aprendizaje (13-28). Disponible en:

http://www.dgdc.unam.mx/Assets/pdfs/sem mar08 2.pdf. [2010,5 de noviembre]

16. Canedo, S. (2009).Contribución al estudio del aprendizaje de las ciencias experimentales en la educación infantil: Cambio Conceptual y construcción de modelos científicos Precursores. Tesis doctoral, Universitat de Barcelona Disponible: http://www.tesisenxarxa.net/TESIS UB/AVAILABLE/TDX-0519109114521//07.SPCI BIBLIOGRAFIA.pdf. [2010,10 de noviembre]

17. Carrasco, J. (2012).Una didáctica para hoy. Como enseñar mejor. Madrid: Ediciones Rialp, S.A. Disponible en:

http://books.google.com.co/books?id=|4bsSI5N7dcC\&pg=PA83\&dq=que+es+una+ +estrategias+did\%C3\%A1cticas\&hl=en\&sa=X\&ei=h60JUdPnKoLU9QSRiYCIBw\& sqi=2\&ved=0CCcQ6AEwAA\#v=onepage \&q=que $\% 20$ es $\% 20$ una $\% 20 \% 20$ estrategia s\%20did\%C3\%A1cticas\&f=false 
Edición Extra-Ordinaria. ISSN 2027-1034 P. p 564-581

Memorias del VII Encuentro Nacional de Experiencias en la Enseñanza de la Biología y la Educación Ambiental y II Congreso Nacional de Investigación en la Enseñanza de la Biología

8. Ferrés, J (1994).Televisión y educación .Barcelona: Paidos.

9. Ferrés, J.(1996).Televisión subliminal. Socialización mediante comunicaciones inadvertidas. [en línea]. Barcelona: Paidos. Disponible en:

Http://books.google.com/books?ld=Rb9wPS1 |4UC\&printsec=frontcover\&dq=ioan ++ ferr\%C3\%a9s\&hl=es\&ei=cozztfs6iimetgfbx8h1bg\&sa=X\&oi=book result\&ct=res ult\&resnum=1\&ved=0CC0Q6AEwAA\#v=onepage\&q\&f=false. [2011,7 de octubre]

10. Fernández, I; Gil, D; Carrascosa, J; Cachapuz, A; Praia, J. (2.002). Visiones deformadas de la ciencia transmitidas por la enseñanza. Revista electrónica enseñanza de las ciencias 20(3),477-488. Disponible en:

http://ddd.uab.es/pub/edlc/02124521v20n3p477.pdf

11. Sanmartí, N. (2002). didáctica de las ciencias en la educación secundaria obligatoria. Madrid: Síntesis educación.

12. Jiménez, M; De Manuel, E; Gonzales; Salinas, F (2.000). La utilización del concepto de ph en la publicidad y su relación con las ideas que manejan los alumnos: aplicaciones en el aula. Revista electrónica de enseñanza de las ciencias, 18(3) 451-461. Disponible en:

http://www.raco.cat/index.php/ensenanza/article/viewFile/21696/21530 